\title{
Asymptotically Almost Periodic Solutions for a Class of Stochastic Functional Differential Equations
}

\author{
Aimin Liu, ${ }^{1}$ Yongjian Liu, ${ }^{2}$ and Qun Liu ${ }^{2}$ \\ ${ }^{1}$ Educational Technology Center, Yulin Normal University, Yulin 537000, China \\ ${ }^{2}$ School of Mathematics and Information Science, Yulin Normal University, Yulin 537000, China \\ Correspondence should be addressed to Yongjian Liu; liuyongjianmaths@126.com
}

Received 7 February 2014; Revised 29 March 2014; Accepted 29 March 2014; Published 6 May 2014

Academic Editor: Yonghui Xia

Copyright (c) 2014 Aimin Liu et al. This is an open access article distributed under the Creative Commons Attribution License, which permits unrestricted use, distribution, and reproduction in any medium, provided the original work is properly cited.

\begin{abstract}
This work is concerned with the quadratic-mean asymptotically almost periodic mild solutions for a class of stochastic functional differential equations $\mathrm{d} x(t)=\left[A(t) x(t)+F\left(t, x(t), x_{t}\right)\right] \mathrm{d} t+H\left(t, x(t), x_{t}\right) \circ \mathrm{d} W(t)$. A new criterion ensuring the existence and uniqueness of the quadratic-mean asymptotically almost periodic mild solutions for the system is presented. The condition of being uniformly exponentially stable of the strongly continuous semigroup $\{T(t)\}_{t \geq 0}$ is essentially removed, which is generated by the linear densely defined operator $A: D(A) \subset L^{2}(\mathbb{P}, \mathbb{U}) \rightarrow L^{2}(\mathbb{P}, \mathbb{H})$, only using the exponential trichotomy of the system, which reflects a deeper analysis of the behavior of solutions of the system. In this case the asymptotic behavior is described through the splitting of the main space into stable, unstable, and central subspaces at each point from the flow's domain. An example is also given to illustrate our results.
\end{abstract}

\section{Introduction}

The theory of almost periodic functions was first developed by the Danish mathematician H. Bohr in 1925-1926. Then Bohr's work was developed substantially by S. Bochner, J. Favard, V. V. Stepanov, and others. Generalization of the classical theory of almost periodic functions has been taken in several directions. These works were recapitulated in literatures [1] and [2]. The concept of almost periodicity is important in probability for investigating stochastic processes [3-7]. Such a notion is also of interest for applications arising in mathematical physics and statistics. Literature [8] developed the notion of $p$-mean almost periodicity based on the concept of quadratic mean uniformly almost periodic utilized by [7] and pointed out that each $p$-mean almost periodic process is uniformly continuous and stochastically bounded [9]. Literature [8] also pointed that the collection of all $p$-mean almost periodic processes is a Banach space when it is equipped with some norm obtained through the norm of $L^{p}(\mathbb{P}, \mathbb{B})$, where $(\mathbb{B},\|\cdot\|)$ is a Banach space.

The asymptotically almost periodic functions were first introduced by Fréchet. In the modern theory of differential equations, many authors [1,2] applied successfully the asymptotic property to determine the existence of almost periodic solutions. Along with the development of such equations as the evolution partial differential equations, functional differential equations, and so forth, where the phase spaces are infinite, the theory of Banach valued asymptotically almost periodic functions had been developed [10-12]. Some techniques in functional analysis and harmonic analysis were applied to such equations; for example, in $[1,13]$, the authors applied spectrum theory to get almost periodic solutions for some linear abstract evolution differential equations. More recently, [14] developed the notion of $p$-mean asymptotical almost periodicity for stochastic processes. Among others, it showed that each $p$-mean asymptotically almost periodic stochastic process is stochastically bounded.

Recently, [8] studied the existence and uniqueness of quadratic-mean almost periodic solutions for the class of stochastic differential equations

$$
\begin{array}{r}
\mathrm{d} x(t)=A x(t) \mathrm{d} t+F(t, x(t)) \mathrm{d} t+G(t, x(t)) \circ \mathrm{d} W(t), \\
t \in \mathbb{R} .
\end{array}
$$


Literature [15] investigated the existence and stability of quadratic-mean almost periodic mild solutions for stochastic functional differential equations

$$
\begin{aligned}
\mathrm{d} x(t)= & {\left[A x(t)+F\left(t, x(t), x_{t}\right)\right] \mathrm{d} t } \\
+ & G\left(t, x(t), x_{t}\right) \circ \mathrm{d} W(t), \quad t \in[0, T], \\
& x(t)=\varphi(t), \quad t \in[-\sigma, 0] .
\end{aligned}
$$

They both assumed that the strongly continuous semigroup $\{T(t)\}_{t \geq 0}$ is uniformly exponentially stable, which is generated by the linear densely defined operator $A: D(A) \quad C$ $L^{2}(\mathbb{P}, \mathbb{H}) \rightarrow L^{2}(\mathbb{P}, \mathbb{H})$. For other works, we refer the reader to $[16-21]$ and the references therein.

One should point that the following condition (C) is very much important in the above-mentioned literatures.

(C) The operator $A$ is the generator of a uniformly exponentially stable semigroup $\{T(t)\}_{t \geq 0}$ such that there exist constants $M>0, \delta>0$ with $\|T(t)\| \leq$ $M e^{-\delta t},(t \geq 0)$.

It is clear that the condition (C) is too strict [22] so that it cannot be satisfied even if for simple $A=\operatorname{diag}\{1,-1\}$ or $A=$ $\operatorname{diag}\{1,0,-1\}$. Literature [22] presented some new criteria ensuring the existence and uniqueness of quadratic-mean almost periodic solution for stochastic differential equation (1), and only assumes that the linear system

$$
\mathrm{d} x(t)=A(t) \mathrm{d} t
$$

admits exponential dichotomy. It is clear that when $A(t)=$ $\operatorname{diag}\{1,-1\}$, the system (3) admits exponential dichotomy. More generally, in the case $A(t) \equiv A$, a constant matrix, the system (3) admits exponential dichotomy if and only if the eigenvalues of $A$ have a nonzero real part. Literature $[14,17]$ has obtained the existence and uniqueness of quadratic-mean almost automorphic solutions or asymptotically almost periodic solutions for stochastic functional differential equations under a hyperbolic and analytic semigroup $\{T(t)\}_{t \geq 0}$. At the same time, one notices that the case that the eigenvalues of $A$ have a zero real part is very common; for example, $A(t)=\operatorname{diag}\{1,0,-1\}$. Therefore, it is interesting to ask, what is that, when the semigroup $\{T(t)\}_{t \geq 0}$ is not exponentially stable, which is generated by the family $\{A(t): t \in \mathbb{R}\}$, or when the semigroup $\{T(t)\}_{t \geq 0}$ is nonhyperbolic? This question will be considered in the paper.

In the present paper, motivated by $[8,14,15]$, we discuss the existence and uniqueness of quadratic-mean asymptotically almost periodic solution to the following stochastic functional differential equation on $L^{2}(\mathbb{P}, \mathbb{M})$, where $\mathbb{P}$ is the probability measure of the probability space $(\Omega, \mathscr{F}, \mathbb{P})$ and $(\mathbb{H},\|\cdot\|)$ is a real separable Hilbert space:

$$
\begin{aligned}
\mathrm{d} x(t)= & {\left[A(t) x(t)+F\left(t, x(t), x_{t}\right)\right] \mathrm{d} t } \\
& +H\left(t, x(t), x_{t}\right) \circ \mathrm{d} W(t), \quad t \in \mathbb{R} .
\end{aligned}
$$

We present the new criterion ensuring the existence of a unique quadratic-mean asymptotically almost periodic solution for the stochastic functional differential equation (1), by employing the properties of almost periodic function and the technique of inequality. We essentially remove the above conditions (C) and only assume that the linear system (2) admits exponential trichotomy (see Definition 11). We also point out that exponential trichotomy is the most complex asymptotic property of dynamical systems arising from the central manifold theory. Starting from the idea that the center manifold of an equilibrium point of a dynamical system consists of orbits whose behavior around the equilibrium point is not controlled by either the attraction of the stable manifold or the repulsion of the unstable manifold. The exponential trichotomy reflects a deeper analysis of the behavior of solutions of dynamical systems. In this case the asymptotic behavior is described through the splitting of the main space into stable, unstable, and central subspaces at each point from the flow's domain.

This paper is organized as follows. In Section 2, the relating notations, definitions, and the basic results are introduced, which would be used later. In Section 3, a new criterion ensuring the existence and uniqueness of a quadratic-mean asymptotically almost periodic mild solution for stochastic functional differential equations is presented. In Section 4, an example is given to illustrate our results. Finally, conclusions are drawn in Section 5.

\section{Preliminaries}

Let $(\Omega, \mathscr{F}, \mathbb{P})$ be a probability space, for a Banach space $(\mathbb{B}, \| \cdot$ $\|)$ and $p \geq 1$, denoted by $L^{p}(\mathbb{P}, \mathbb{B})$, the Banana space of all $\mathbb{B}$-value random variable $x$, such that

$$
\mathbb{E}\|x\|^{p}=\int_{\Omega}\|x\|^{p} \mathrm{~d} \mathbb{P}<\infty .
$$

It is then routine to check that $L^{p}(\mathbb{P}, \mathbb{B})$ is a Banach space when it is equipped with its natural norm $\|\cdot\|_{p}$ defined by

$$
\|x\|_{L^{p}(\mathbb{P}, \mathbb{B})}=\left(\int_{\Omega}\|x\|^{p} \mathrm{~d} \mathbb{P}\right)^{1 / p}
$$

for each $x \in L^{p}(\mathbb{P}, \mathbb{B})$.

This setting requires the following preliminary definitions.

Definition 1. A stochastic process $x: \mathbb{R} \rightarrow L^{p}(\mathbb{P}, \mathbb{B})$ is said to be continuous whenever

$$
\lim _{t \rightarrow s} \mathbb{E}\|x(t)-x(s)\|^{p}=0 .
$$

Definition 2. A stochastic process $x: \mathbb{R} \rightarrow L^{p}(\mathbb{P}, \mathbb{B})$ is said to be stochastically bounded whenever

$$
\lim _{N \rightarrow \infty} \sup _{t \in \mathbb{R}} \mathbb{P}\{\|x(t)\|>N\}=0 .
$$

Let $\operatorname{CUB}\left(\mathbb{R} ; L^{p}(\mathbb{P}, \mathbb{B})\right)$ denote the collection of all stochastic processes $x: \mathbb{R} \rightarrow L^{p}(\mathbb{P}, \mathbb{B})$, which are continuous and uniformly bounded. It is easily to check that $\operatorname{CUB}\left(\mathbb{R} ; L^{p}(\mathbb{P}, \mathbb{B})\right)$ is a Banach space when it is equipped with the norm

$$
\|x\|_{\infty}=\sup _{t \in \mathbb{R}}\left(\mathbb{E}\|x(t)\|^{p}\right)^{1 / p}
$$


Definition 3. A continuous stochastic process $x: \mathbb{R} \rightarrow$ $L^{p}(\mathbb{P}, \mathbb{B})$ is said to be $p$-mean almost periodic if for each $\varepsilon>0$ there exists $l(\varepsilon)>0$ such that any interval of length $l(\varepsilon)$ contains at least a number $\tau$ for which

$$
\sup _{t \in \mathbb{R}} \mathbb{E}\|x(t+\tau)-x(t)\|^{p}<\varepsilon .
$$

The collection of all stochastic processes $x: \mathbb{R} \rightarrow$ $L^{p}(\mathbb{P}, \mathbb{B})$ which are $p$-mean almost periodic is denoted by $A P\left(\mathbb{R} ; L^{p}(\mathbb{P}, \mathbb{B})\right) . A P\left(\mathbb{R} ; L^{p}(\mathbb{P}, \mathbb{B})\right)$ is a closed subspace of $\operatorname{CUB}\left(\mathbb{R} ; L^{p}(\mathbb{P}, \mathbb{B})\right)$. Therefore, $A P\left(\mathbb{R} ; L^{p}(\mathbb{P}, \mathbb{B})\right)$ is a Banach space when it is equipped with the norm $\|\cdot\|_{\infty}$ (see, e.g., [3]).

Let $\left(\mathbb{B}_{1},\|\cdot\|_{1}\right),\left(\mathbb{B}_{2},\|\cdot\|_{2}\right)$ be two Banach spaces and $L^{p}\left(\mathbb{P}, \mathbb{B}_{1}\right), L^{p}\left(\mathbb{P}, \mathbb{B}_{2}\right)$ their corresponding $L^{p}$-spaces, respectively.

Definition 4 (see [8]). A function $f: \mathbb{R} \times L^{p}\left(\mathbb{P}, \mathbb{B}_{1}\right) \rightarrow$ $L^{p}\left(\mathbb{P}, \mathbb{B}_{2}\right),(t, x) \rightarrow f(t, x)$, which is jointly continuous, is said to be $p$-mean almost periodic in $t \in \mathbb{R}$ uniformly in $x \in \mathbb{K}$ where $\mathbb{K} \subset L^{p}\left(\mathbb{P}, \mathbb{B}_{1}\right)$ is compact if, for any $\varepsilon>0$, there exists $l(\varepsilon, \mathbb{K})>0$ such that any interval of length $l(\varepsilon, \mathbb{K})$ contains at least a number $\tau$ for which

$$
\sup _{t \in \mathbb{R}}\left(\mathbb{E}\|f(t+\tau, x)-f(t, x)\|^{p}\right)^{1 / p}<\varepsilon
$$

for each stochastic process $x: \mathbb{R} \rightarrow \mathbb{K}$.

Theorem 5. Let $f: \mathbb{R} \times L^{p}\left(\mathbb{P}, \mathbb{B}_{1}\right) \rightarrow L^{p}\left(\mathbb{P}, \mathbb{B}_{2}\right),(t, x) \rightarrow$ $f(t, x)$, be a p-mean almost periodic process in $t \in \mathbb{R}$ uniformly in $x \in \mathbb{K}$, where $\mathbb{K} \subset L^{p}\left(\mathbb{P}, \mathbb{B}_{1}\right)$ is compact. Then for every real sequence $\alpha^{\prime}$, there exists $\beta \subset \alpha^{\prime}$ and a continuous function $g(t, x)$ such that $T_{\beta} f(t, x)=g(t, x)$ exists uniformly on $\mathbb{R} \times \mathbb{K}$. Further, $g(t, x)$ is also $p$-mean almost periodic process in $t \in \mathbb{R}$ uniformly in $x \in \mathbb{K}$.

The space of the restrictions of all $p$-mean almost periodic stochastic processes on $\mathbb{R}$ is denoted by $A P\left(\mathbb{R} ; L^{P}(\mathbb{P}, \mathbb{B})\right)$, and $p$-mean almost periodic stochastic processes in $t$, uniformly for $x$ in compact subset $\mathbb{K}$ of $L^{p}\left(\mathbb{P}, \mathbb{B}_{1}\right)$ by $A P(\mathbb{R} \times$ $\left.L^{p}\left(\mathbb{P}, \mathbb{B}_{1}\right) ; L^{p}\left(\mathbb{P}, \mathbb{B}_{2}\right)\right)$.

Denote by $C_{0}\left(\mathbb{R} ; L^{p}(\mathbb{P}, \mathbb{B})\right)$ the space of all continuous stochastic processes $Z: \mathbb{R} \rightarrow L^{p}(\mathbb{P}, \mathbb{B})$ such that $\lim _{t \rightarrow+\infty} \mathbb{E}$ $\|Z(t)\|^{p}=0$, and denote by $C_{0}\left(\mathbb{R} \times L^{p}\left(\mathbb{P}, \mathbb{B}_{1}\right) ; L^{p}\left(\mathbb{P}, \mathbb{B}_{2}\right)\right)$ the space of all continuous functions $Z: \mathbb{R} \times L^{p}\left(\mathbb{P}, \mathbb{B}_{1}\right) \rightarrow$ $L^{p}\left(\mathbb{P}, \mathbb{B}_{2}\right)$ such that $\lim _{t \rightarrow+\infty} \mathbb{E}\|Z(t, x)\|_{2}^{p}=0$ uniformly for $x$ in any compact subset of $L^{p}\left(\mathbb{P}, \mathbb{B}_{1}\right)$.

Definition 6. A stochastic process $X: R \rightarrow L^{p}(\mathbb{P}, \mathbb{B})$ is called $p$-mean asymptotically almost periodic if there exist two stochastic processes $Y \in A P\left(\mathbb{R} ; L^{p}(\mathbb{P}, \mathbb{B})\right)$ and $Z \in$ $C_{0}\left(\mathbb{R} ; L^{p}(\mathbb{P}, \mathbb{B})\right)$ such that $X=Y+Z$.

By $A A P\left(\mathbb{R} ; L^{p}(\mathbb{P}, \mathbb{B})\right)$ one denotes the collection of all $p$ mean asymptotically almost periodic stochastic processes.

Definition 7. A stochastic process $X: R \times L^{p}\left(\mathbb{P}, \mathbb{B}_{1}\right) \rightarrow L^{p}$ $\left(\mathbb{P}, \mathbb{B}_{2}\right)$ is called $p$-mean asymptotically periodic if there exist two stochastic processes $Y \in A P\left(\mathbb{R} \times L^{p}\left(\mathbb{P}, \mathbb{B}_{1}\right) ; L^{p}\left(\mathbb{P}, \mathbb{B}_{2}\right)\right)$ and $Z \in C_{0}\left(\mathbb{R} \times L^{p}\left(\mathbb{P}, \mathbb{B}_{1}\right) ; L^{p}\left(\mathbb{P}, \mathbb{B}_{2}\right)\right)$ such that $X=Y+Z$.
By $A A P\left(\mathbb{R} \times L^{p}\left(\mathbb{P}, \mathbb{B}_{1}\right) ; L^{p}\left(\mathbb{P}, \mathbb{B}_{2}\right)\right)$ we denote the collection of such function.

Lemma 8 (see $[14])$. If $X$ belongs to $A A P\left(\mathbb{R} ; L^{p}(\mathbb{P}, \mathbb{B})\right)$, one has the following:

(1) there exists a constant $M>0$ such that $\mathbb{E}\|X(t)\|^{p} \leq M$ for each $t \in \mathbb{R}$;

(2) $X$ is stochastically bounded.

It is easy to see that $A A P\left(\mathbb{R} ; L^{p}(\mathbb{P}, \mathbb{B})\right)=A P\left(\mathbb{R} ; L^{p}(\mathbb{P}, \mathbb{B})\right)$ $\oplus C_{0}\left(\mathbb{R} ; L^{p}(\mathbb{P}, \mathbb{B})\right)$. Then the space $A A P\left(\mathbb{R} ; L^{p}(\mathbb{P}, \mathbb{B})\right)$ of $p$ mean asymptotically almost periodic stochastic processes is a Banach space when it is equipped with the norm $\|\cdot\|_{\infty}$.

Lemma 9 (see [14]). Let $x \in A P\left(\mathbb{R}_{+} ; L^{p}\left(\mathbb{P}, \mathbb{B}_{1}\right)\right), K=$ $\overline{\left\{\left(x(t), x_{t}\right): t \in \mathbb{R}_{+}\right\}}$, where $x_{t}=\{x(t+h):-\sigma<h<0\}$ is regarded as a $C\left([-\sigma, 0], L^{p}\left(\mathbb{P}, \mathbb{B}_{1}\right)\right)$ valued stochastic process. Moreover

$$
\begin{aligned}
f & \in A P\left(\mathbb{R}_{+} \times L^{p}\left(\mathbb{P}, \mathbb{B}_{1}\right)\right) \\
& \times C\left([-\sigma, 0], L^{p}\left(\mathbb{P}, \mathbb{B}_{1}\right) ; L^{p}\left(\mathbb{P}, \mathbb{B}_{2}\right)\right), \\
f & \in C_{K}\left(\mathbb{R}_{+} \times L^{p}\left(\mathbb{P}, \mathbb{B}_{1}\right)\right) \\
& \times C\left([-\sigma, 0], L^{p}\left(\mathbb{P}, \mathbb{B}_{1}\right) ; L^{p}\left(\mathbb{P}, \mathbb{B}_{2}\right)\right) .
\end{aligned}
$$

Then $f(\cdot, x(\cdot), x) \in A P\left(\mathbb{R}_{+} ; L^{p}\left(\mathbb{P}, \mathbb{B}_{2}\right)\right)$.

Lemma 10 (see [14]). Let $x \in A A P\left(\mathbb{R}_{+} ; L^{p}\left(\mathbb{P}, \mathbb{B}_{1}\right)\right), K=$ $\overline{\left\{\left(x(t), x_{t}\right): t \in \mathbb{R}_{+}\right\}}$, where $x_{t}=\{x(t+h):-\sigma<h<0\}$ is regarded as a $C\left([-\sigma, 0], L^{p}\left(\mathbb{P}, \mathbb{B}_{1}\right)\right)$ valued stochastic process. Moreover

$$
\begin{aligned}
f & \in A A P\left(\mathbb{R}_{+} \times L^{p}\left(\mathbb{P}, \mathbb{B}_{1}\right)\right) \\
& \times C\left([-\sigma, 0], L^{p}\left(\mathbb{P}, \mathbb{B}_{1}\right) ; L^{p}\left(\mathbb{P}, \mathbb{B}_{2}\right)\right), \\
f & \in C_{K}\left(\mathbb{R}_{+} \times L^{p}\left(\mathbb{P}, \mathbb{B}_{1}\right)\right) \\
& \times C\left([-\sigma, 0], L^{p}\left(\mathbb{P}, \mathbb{B}_{1}\right) ; L^{p}\left(\mathbb{P}, \mathbb{B}_{2}\right)\right) .
\end{aligned}
$$

Then $f(\cdot, x(\cdot), x) \in A A P\left(\mathbb{R}_{+} ; L^{p}\left(\mathbb{P}, \mathbb{B}_{2}\right)\right)$.

Next, one introduces a crucial concept [23].

Suppose that $U(t)$ is the fundamental matrix solution of the linear differential system

$$
\frac{\mathrm{d} x}{\mathrm{~d} t}=A(t) x
$$

where $A(t)$ is a linear continuous operator, with $U(0)=I$.

Definition 11 (see [23]). System (14) is said to admit exponential trichotomy if there are linear projections $P, Q$ such that

$$
P Q=Q P, \quad P+Q-P Q=I,
$$


and constants $\alpha>0$ and $\beta \geq 1$ such that

$$
\begin{array}{r}
\left\|U(t) P U^{-1}(s)\right\|_{p} \leq \beta \exp (-\alpha(t-s)), \\
\text { for } 0 \leq s \leq t, \\
\left\|U(t)(I-P) U^{-1}(s)\right\|_{p} \leq \beta \exp (-\alpha(s-t)), \\
\quad \text { for } t \leq s, \quad s \geq 0, \\
\left\|U(t) Q U^{-1}(s)\right\|_{p} \leq \beta \exp (-\alpha(s-t)), \\
\quad \text { for } t \leq s \leq 0, \\
\left\|U(t)(I-Q) U^{-1}(s)\right\|_{p} \leq \beta \exp (-\alpha(t-s)), \\
\text { for } s \leq t, \quad s \leq 0 .
\end{array}
$$

If in the above definition we put $Q=I-P,(16)$ becomes

$$
\begin{gathered}
\left\|U(t) P U^{-1}(s)\right\|_{p} \leq \beta \exp (-\alpha(t-s)), \quad \text { for } s \leq t, \\
\left\|U(t)(I-P) U^{-1}(s)\right\|_{p} \leq \beta \exp (\alpha(t-s)), \quad \text { for } t \leq s .
\end{gathered}
$$

It is clear that when $A(t)=-\tanh t$, the system (14) admits exponential trichotomy. More generally, in the case $A(t) \equiv A=\operatorname{diag}\{1,0,-1\}$, a constant matrix, the system (14) admits exponential trichotomy.

Theorem 12. Let $A(t)$ be a continuous linear operator on $\mathbb{R}$ and let (14) have an exponential trichotomy (16) on $\mathbb{R}_{+}$. If for some sequence $a=\left\{a_{n}\right\}, \lim _{n \rightarrow+\infty} a_{n}=+\infty$, $T_{a} A(t)=B(t)$ uniformly on compact subintervals of $\mathbb{R}$, $\lim _{n \rightarrow+\infty} U\left(a_{n}\right) P U^{-1}\left(a_{n}\right)=P^{*}, \lim _{n \rightarrow+\infty} U\left(a_{n}\right) Q U^{-1}\left(a_{n}\right)=$ $Q^{*}$, and the equation (which is called the hull equation (14))

$$
\frac{d y}{d t}=B(t) y
$$

has an exponential trichotomy on $\mathbb{R}$ with projections $P^{*}, Q^{*}$ and the same constants $\alpha, \beta$.

Proof. The translated equation

$$
\frac{\mathrm{d} x}{\mathrm{~d} t}=A\left(t+a_{n}\right) x
$$

has the fundamental matrix

$$
\begin{gathered}
U_{n}(t)=U\left(t+a_{n}\right) U^{-1}\left(a_{n}\right), \\
\left\|U_{n}(t) P_{n} U_{n}^{-1}(s)\right\|_{p} \leq \beta \exp (-\alpha(t-s)),
\end{gathered}
$$

$$
\begin{gathered}
t \geq s \geq-a_{n}, \\
\left\|U_{n}(t)\left(I-P_{n}\right) U_{n}^{-1}(s)\right\|_{p} \leq \beta \exp (\alpha(t-s)), \\
s \geq t \geq-a_{n}, \\
\left\|U_{n}(t) Q_{n} U_{n}^{-1}(s)\right\|_{p} \leq \beta \exp (-\alpha(t-s)), \\
t \leq s \leq a_{n}, \\
\left\|U_{n}(t)\left(I-Q_{n}\right) U_{n}^{-1}(s)\right\|_{p} \leq \beta \exp (\alpha(t-s)),
\end{gathered}
$$

$$
s \leq t \leq a_{n},
$$

where $P_{n}=U\left(a_{n}\right) P U^{-1}\left(a_{n}\right), Q_{n}=U\left(a_{n}\right) Q U^{-1}\left(a_{n}\right)$. Since $\left|P_{n}\right| \leq \beta,\left|Q_{n}\right| \leq \beta$ by restricting attention to a subsequence we can assume that $P_{n} \rightarrow P^{*}, Q_{n} \rightarrow Q^{*}$, where $P^{*}, Q^{*}$ are projections. Since $U_{n}(t) \rightarrow V(t)$ for every $t$, where $V(t)$ is the fundament matrix of (21) such that $V(0)=I$, it follows that

$$
\begin{array}{r}
\left\|V_{n}(t) P^{*} V_{n}^{-1}(s)\right\|_{p} \leq \beta \exp (-\alpha(t-s)), \\
t \geq s \geq 0, \\
\left\|V_{n}(t)\left(I-P^{*}\right) V_{n}^{-1}(s)\right\|_{p} \leq \beta \exp (\alpha(t-s)), \\
s \geq t, \quad s \geq 0, \\
\left\|V_{n}(t) Q^{*} V_{n}^{-1}(s)\right\|_{p} \leq \beta \exp (-\alpha(t-s)), \\
t \leq s \leq 0, \\
\left\|V_{n}(t)\left(I-Q^{*}\right) V_{n}^{-1}(s)\right\|_{p} \leq \beta \exp (\alpha(t-s)), \\
s \geq t, \quad s \leq 0 .
\end{array}
$$

Since the projection corresponding to an exponential trichotomy on $\mathbb{R}$ is uniquely determined it follows that $P_{n} \rightarrow$ $P^{*}, Q_{n} \rightarrow Q^{*}$ without restriction to a subsequence.

\section{Existence of Asymptotically Almost Periodic Solutions}

For convenience, throughout this section, let $(\mathbb{\square},\|\cdot\|)$ be a real separable Hilbert space, and let $(\Omega, \mathscr{F}, \mathbb{P})$ be a complete probability space equipped with a normal filtration $\left\{\mathscr{F}_{t}\right.$ : $t \in \mathbb{R}\}$, that is, a right-continuous, increasing family of sub- $\sigma$-algebras of $\mathscr{F}$. Let $\mathscr{L}\left(L^{2}(\mathbb{P}, \mathbb{H})\right)$ be the space of all bounded linear operators from $L^{2}(\mathbb{P}, \mathbb{U})$ to $L^{2}(\mathbb{P}, \mathbb{U})$ and $C=$ $C\left([-\sigma, 0], L^{2}(\mathbb{P}, \mathbb{M})\right)$ the space of all continuous functions from $[-\sigma, 0]$ to $L^{2}(\mathbb{P}, \mathbb{U})$ with the sup norm

$$
\|\psi\|_{C}=\sup \left\{\|\psi(s)\|_{L^{2}(\mathbb{P}, \mathbb{H})}: \psi \in C,-\sigma \leq s \leq 0\right\} .
$$


For any continuous $\mathscr{F}_{t}$-adapted $L^{2}(\mathbb{P}, \mathbb{U})$-valued stochastic process $x(t): \Omega \rightarrow L^{2}(\mathbb{P}, \mathbb{H}), t \geq-\sigma$, we associate it with a continuous $\mathscr{F}_{t}$-adapted $C$-valued stochastic process $x_{t}: \Omega \rightarrow C, t \geq 0$, by setting $x_{t}(s)(\omega)=x(t+s)(\omega), s \in$ $[-\sigma, 0]$.

In this section, we study the existence and uniqueness of quadratic-mean asymptotically almost periodic mild solution to stochastic functional differential equations of the form

$$
\begin{aligned}
\mathrm{d} x(t)= & {\left[A(t) x(t)+F\left(t, x(t), x_{t}\right)\right] \mathrm{d} t } \\
& +H\left(t, x(t), x_{t}\right) \circ \mathrm{d} W(t), \quad t \in \mathbb{R},
\end{aligned}
$$

where $A(t)$ is a linear operator and $D(A) \subset L^{2}(\mathbb{P}, \mathbb{H}) \rightarrow$ $L^{2}(\mathbb{P}, \mathbb{U})$ generates a strongly continuous semigroup $\{T(t)\}_{t \geq 0}$, which is nonhyperbolic. That is to say, the linear operator $A(t)$ may exhibit central flow. $W(t)$ is a certain $Q$-Wiener process with covariance operator $Q$ and takes

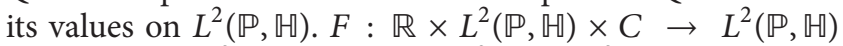
and $H: \mathbb{R} \times L^{2}(\mathbb{P}, \mathbb{W}) \times C \rightarrow L^{2}\left(\mathbb{P}, \mathscr{L}\left(L^{2}(\mathbb{P}, \mathbb{R})\right)\right)$ are two continuous mappings.

Throughout this section, we require the following assumptions.

(H1) Suppose that $F \in A A P\left(\mathbb{R} \times L^{2}(\mathbb{P}, \mathbb{a}) \times C ; L^{2}(\mathbb{P}, \mathbb{A})\right)$. Furthermore, there exists a constant $F_{l}>0$ such that

$$
\begin{aligned}
& \left\|F\left(t, x_{1}, x_{2}\right)-F\left(t, y_{1}, y_{2}\right)\right\|_{L^{2}(\mathbb{P}, \mathbb{H})}^{2} \\
& \quad \leq F_{l}\left(\left\|x_{1}-y_{1}\right\|_{L^{2}(\mathbb{P}, \mathbb{H})}^{2}+\left\|x_{2}-y_{2}\right\|_{C}^{2}\right)
\end{aligned}
$$

for all stochastic processes $\left(x_{1}, x_{2}\right),\left(y_{1}, y_{2}\right) \in L^{2}(\mathbb{P}$, $\mathbb{U}$ ) $\times C$ and for each $t \in \mathbb{R}$.

(H2) Suppose that $H \in A A P\left(\mathbb{R} \times L^{2}(\mathbb{P}, \mathbb{1}) \times C ; L^{2}\left(\mathbb{P}, \mathscr{L}\left(L^{2}\right.\right.\right.$

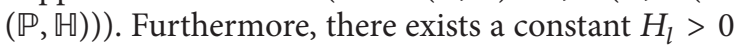
such that

$$
\begin{aligned}
& \left\|H\left(t, x_{1}, x_{2}\right)-H\left(t, y_{1}, y_{2}\right)\right\|_{L^{2}\left(\mathbb{P}, \mathscr{L}\left(L^{2}(\mathbb{P}, \mathbb{H})\right)\right.}^{2} \\
& \quad \leq H_{l}\left(\left\|x_{1}-y_{1}\right\|_{L^{2}(\mathbb{P}, H)}^{2}+\left\|x_{2}-y_{2}\right\|_{C}^{2}\right),
\end{aligned}
$$

for all stochastic processes $\left(x_{1}, x_{2}\right),\left(y_{1}, y_{2}\right) \in$ $L^{2}(\mathbb{P}, \mathbb{U}) \times C$ and for each $t \in \mathbb{R}$.

(H3) Suppose that the linear system of (26)

$$
\mathrm{d} x(t)=A(t) \mathrm{d} t
$$

admits exponential trichotomy (see Definition 11); that is, there exist constants $\alpha>0, \beta \geq 1$ such that (16) holds.

Define the function $G(t, s)$ as the form

$$
G(t, s)= \begin{cases}U(t) P U^{-1}(s), & 0 \leq s \leq t, \\ -U(t)(I-P) U^{-1}(s), & \max \{t, 0\} \leq s, \\ -U(t) Q U^{-1}(s), & 0 \geq s \geq t, \\ U(t)(I-Q) U^{-1}(s), & \min \{t, 0\} \geq s,\end{cases}
$$

where $U(t)$ is the fundamental matrix solution of the linear differential system (29) with $U(0)=I$.
Definition 13. A $\mathscr{F}_{t}$-progressive process $\{x(t)\}_{t \in \mathbb{R}}$ is called a mild solution of (26) on $\mathbb{R}$ if

$$
\begin{aligned}
x(t)= & G(t, s) x(s)+\int_{s}^{t} G(t, \tau) F\left(\tau, x(\tau), x_{\tau}\right) \mathrm{d} \tau \\
& +\int_{s}^{t} G(t, \tau) H\left(\tau, x(\tau), x_{\tau}\right) \circ \mathrm{d} W(\tau),
\end{aligned}
$$

for all $t \geq s$, for each $s \in \mathbb{R}$, where $G(t, s)$ is defined by (30).

Theorem 14. Assume that conditions (H1)-(H3) are satisfied. And the positive constants $\alpha, \beta, F_{l}$, and $H_{l}$ satisfy the following condition:

$$
\Lambda=\frac{8 \beta}{\alpha} \sqrt{F_{l}+\frac{\alpha H_{l}}{2}}<1 .
$$

Then the system (26) has a unique quadratic-mean asymptotically almost periodic mild solution, which can be explicitly expressed as follows:

$$
x(t)=\sum_{i=1}^{4} \Phi^{i} x(t)+\sum_{i=1}^{4} \Psi^{i} x(t),
$$

where

$$
\begin{aligned}
& \Phi^{1} x(t):=\int_{0}^{t} U(t) P U^{-1}(s) F\left(s, x(s), x_{s}\right) d s, \\
& \Phi^{2} x(t):=\int_{+\infty}^{\max \{t, 0\}} U(t)(I-P) U^{-1}(s) F\left(s, x(s), x_{s}\right) d s, \\
& \Phi^{3} x(t):=\int_{0}^{t} U(t) Q U^{-1}(s) F\left(s, x(s), x_{s}\right) d s, \\
& \Phi^{4} x(t):=\int_{-\infty}^{\min \{t, 0\}} U(t)(I-Q) U^{-1}(s) F\left(s, x(s), x_{s}\right) d s, \\
& \Psi^{1} x(t):=\int_{0}^{t} U(t) P U^{-1}(s) H\left(s, x(s), x_{s}\right) \circ d W(s), \\
& \Psi^{2} x(t):=\int_{+\infty}^{\max \{t, 0\}} U(t)(I-P) U^{-1}(s) \\
& \Psi^{3} x(t):=\int_{0}^{t} U(t) Q U^{-1}(s) H\left(s, x(s), x_{s}\right) \circ d W(s), \\
& \Psi^{4} x(t):=\int_{-\infty}^{\min \{t, 0\}} U(t)(I-Q) U^{-1}(s) \\
& \quad \times H\left(s, x(s), x_{s}\right) \circ d W(s) .
\end{aligned}
$$

We show that (26) exists as a mild solution. Note that (33) and (34) are well defined for each $t \in \mathbb{R}$ and satisfy (31) for all $t \geq s$, for each $s \in \mathbb{R}$. Hence $x(t)$ given by (33) and (34) is a mild solution of (26). 
Define a mapping $\mathbf{L}$ on $C\left(\mathbb{R}, L^{2}(\mathbb{P}, \mathbb{U})\right)$ by

$$
(\mathbf{L} x)(t)=\sum_{i=1}^{4} \Phi^{i} x(t)+\sum_{i=1}^{4} \Psi^{i} x(t):=\left(B_{1} x\right)(t)+\left(B_{2} x\right)(t),
$$

where the $\Phi^{i}, \Psi^{i}$ are defined in (34).

In order to prove Theorem 14, we first prove Lemmas 15 and 16.

Lemma 15. Assume that conditions (H1)-(H3) are satisfied. Then the operator $\mathbf{L}$ maps $A A P\left(\mathbb{R}, L^{2}(\mathbb{P}, \mathbb{E})\right)$ into itself.

Proof. First, let us show that $B_{1} x$ is quadratic-mean asymptotically almost periodic whenever $x \in A A P\left(\mathbb{R}, L^{2}(\mathbb{P}, \mathbb{R})\right)$ dose.

Indeed, assuming that $x$ is quadratic-mean asymptotically almost periodic and using condition (H1) and Lemma 10 , one can easily see that $s \mapsto F\left(s, x(s), x_{s}\right)$ is quadratic-mean asymptotically almost periodic. Therefore, there exist an almost periodic stochastic process $k\left(s, x(s), x_{s}\right)$ and a stochastic process $h \in C_{0}\left(\mathbb{R} \times L^{2}(\mathbb{P}, \mathbb{U}), L^{2}(\mathbb{P}, \mathbb{U})\right)$ such that $F=k+h$. Furthermore, one observes that

$$
\begin{aligned}
\Phi^{1} x(t):= & \int_{0}^{t} U(t) P U^{-1}(s) k\left(s, x(s), x_{s}\right) \mathrm{d} s \\
& +\int_{0}^{t} U(t) P U^{-1}(s) h\left(s, x(s), x_{s}\right) \mathrm{d} s \\
:= & \alpha(t)+\beta(t) .
\end{aligned}
$$

We claim that $\alpha(t) \in A P\left(\mathbb{R}, L^{2}(\mathbb{P}, \mathbb{G})\right)$. In fact, $s \mapsto k(s$, $\left.x(s), x_{s}\right)$ is quadratic-mean almost periodic. Therefore, one can find a common subsequence $\left\{\alpha_{n}\right\}$. One still denotes it as $\alpha=\left\{\alpha_{n}\right\}$, such that

$$
T_{\alpha} k\left(t, x(t), x_{t}\right)=k_{1}\left(t, x(t), x_{t}\right),
$$

uniformly for $t \in \mathbb{R}$, and

$$
\begin{array}{r}
\lim _{k \rightarrow \infty} U\left(t+\alpha_{k}\right) P U^{-1}\left(s+\alpha_{k}\right)=V(t) P^{*} V^{-1}(s), \\
0 \leq s \leq t,
\end{array}
$$

$$
\begin{gathered}
\lim _{k \rightarrow \infty} U\left(t+\alpha_{k}\right)(I-P) U^{-1}\left(s+\alpha_{k}\right)=V(t)\left(I-P^{*}\right) V^{-1}(s), \\
\max \{t, 0\} \leq s, \\
\lim _{k \rightarrow \infty} U\left(t+\alpha_{k}\right) Q U^{-1}\left(s+\alpha_{k}\right)=V(t) Q^{*} V^{-1}(s), \\
0 \geq s \geq t,
\end{gathered}
$$

$\lim _{k \rightarrow \infty} U\left(t+\alpha_{k}\right)(I-Q) U^{-1}\left(s+\alpha_{k}\right)=V(t)\left(I-Q^{*}\right) V^{-1}(s)$, in the sense of norm $\|\cdot\|_{L^{2}(\mathbb{P}, \mathbb{H})}=\left(\int_{\Omega}\|\cdot\|^{2} \mathrm{~d} \mathbb{P}\right)^{1 / 2}$ uniformly for $t \in \mathbb{R}$. Then

$$
\begin{aligned}
& \alpha\left(t+\alpha_{k}\right) \\
& =\int_{0}^{t+\alpha_{k}} U\left(t+\alpha_{k}\right) P U^{-1}(s) k\left(s, x(s), x_{s}\right) \mathrm{d} s \\
& =\int_{0}^{t} U\left(t+\alpha_{k}\right) P U^{-1}\left(s+\alpha_{k}\right) k\left(s+\alpha_{k}, x\left(s+\alpha_{k}\right), x_{s+\alpha_{k}}\right) \mathrm{d} s .
\end{aligned}
$$

From (37), (38), (39), and Lebesgue's control convergence theorem, one sees that $\alpha\left(t+\alpha_{k}\right)$ converges to

$$
\int_{0}^{t} V(t) P^{*} V^{-1}(s) k_{1}\left(s, x(s), x_{s}\right) \mathrm{d} s
$$

uniformly for $t \in \mathbb{R}$. Hence $\alpha(t) \in A P\left(\mathbb{R}, L^{2}(\mathbb{P}, \mathbb{E})\right)$.

Next, let us show that $\beta(t) \in C_{0}\left(\mathbb{R}, L^{2}(\mathbb{P}, \mathbb{H})\right)$. In fact

$$
\begin{aligned}
& \mathbb{E}\|\beta(t)\|^{2} \\
& =\mathbb{E}\left\|\int_{0}^{t} U(t) P U^{-1}(s) h\left(s, x(s), x_{s}\right) \mathrm{d} s\right\|^{2} \\
& \leq \beta^{2} \mathbb{E}\left(\int_{0}^{t} e^{-\alpha(t-s)}\left\|h\left(s, x(s), x_{s}\right)\right\| \mathrm{d} s\right)^{2} \\
& =\beta^{2} \mathbb{E}\left(\int_{0}^{t} e^{-(\alpha(t-s)) / 2} e^{-(\alpha(t-s)) / 2}\left\|h\left(s, x(s), x_{s}\right)\right\| \mathrm{d} s\right)^{2} \\
& \leq \beta^{2} \mathbb{E}\left[\left(\int_{0}^{t} e^{-\alpha(t-s)} \mathrm{d} s\right)\left(\int_{0}^{t} e^{-\alpha(t-s)}\left\|h\left(s, x(s), x_{s}\right)\right\|^{2} \mathrm{~d} s\right)\right] \\
& =\beta^{2}\left(\int_{0}^{t} e^{-\alpha(t-s)} \mathrm{d} s\right)\left(\int_{0}^{t} e^{-\alpha(t-s)} \mathbb{E}\left\|h\left(s, x(s), x_{s}\right)\right\|^{2} \mathrm{~d} s\right) \\
& \leq \beta^{2}\left(\int_{0}^{t} e^{-\alpha(t-s)} \mathrm{d} s\right)^{2} \sup _{t \in \mathbb{R}} \mathbb{E}\left\|h\left(t, x(t), x_{t}\right)\right\|^{2} \\
& =\frac{\beta^{2}}{\alpha^{2}}\left(1-e^{-\alpha t}\right)^{2} \sup _{t \in \mathbb{R}} \mathbb{E}\left\|h\left(t, x(t), x_{t}\right)\right\|^{2} .
\end{aligned}
$$

Since $h \in C_{0}\left(\mathbb{R} \times L^{2}(\mathbb{P}, \mathbb{W}), L^{2}(\mathbb{P}, \mathbb{H})\right)$, we deduce that $\lim _{t \rightarrow+\infty} \mathbb{E}\|\beta(t)\|^{2}=0$.

Therefore, $\Phi^{1} x(\cdot)$ is quadratic-mean asymptotically almost periodic.

Similar to the proof given for $\Phi^{1} x(\cdot)$, one can prove that $\Phi^{2} x(\cdot), \Phi^{3} x(\cdot)$, and $\Phi^{4} x(\cdot)$ are quadratic-mean asymptotically almost periodic.

Thus, $B_{1} x$ is quadratic-mean asymptotically almost periodic whenever $x \in A A P\left(\mathbb{R}, L^{2}(\mathbb{P}, \mathbb{R})\right)$.

Secondly, we show that $B_{2} x$ is also quadratic-mean asymptotically almost periodic whenever $x \in A A P\left(\mathbb{R}, L^{2}\right.$ $(\mathbb{P}, \mathbb{M})$ ).

Of course, this is more complicated than the previous case because of the involvement of the Brownian motion $W$. 
To overcome such a difficulty, one makes extensive use of the Itô isometry identity and the properties of $\widetilde{W}$ defined by $\widetilde{W} \equiv W\left(s+\alpha_{k}\right)$ for each $s$. Note that $\widetilde{W}$ is also a Brownian motion and has the same distribution as $W$. Assuming that $x$ is quadratic-mean asymptotically almost periodic, using $(\mathrm{H} 2)$ and Lemma 10, one can easily see that $s \mapsto H\left(s, x(s), x_{s}\right)$ is quadratic-mean asymptotically almost periodic. Therefore, there exist an almost periodic stochastic process $n\left(s, x(s), x_{s}\right)$ and a stochastic process $m \in C_{0}\left(\mathbb{R} \times L^{2}(\mathbb{P}, \mathbb{U}), L^{2}(\mathbb{P}, \mathbb{W})\right)$ such that $H=n+m$. Furthermore, one observes that

$$
\begin{aligned}
\Psi^{1} x(t) \equiv & \int_{0}^{t} U(t) P U^{-1}(s) n\left(s, x(s), x_{s}\right) \circ \mathrm{d} W(s) \\
& +\int_{0}^{t} U(t) P U^{-1}(s) m\left(s, x(s), x_{s}\right) \circ \mathrm{d} W(s) \\
:= & N(t)+M(t) .
\end{aligned}
$$

We claim that $N(t) \in A P\left(\mathbb{R}, L^{2}(\mathbb{P}, \mathbb{U})\right)$. In fact, $s \mapsto$ $n\left(s, x(s), x_{s}\right)$ is quadratic-mean almost periodic. Therefore, one can find a common subsequence $\left\{\alpha_{n}\right\}$. One still denotes it as $\alpha=\left\{\alpha_{n}\right\}$, such that

$$
T_{\alpha} n\left(t, x(t), x_{t}\right)=n_{1}\left(t, x(t), x_{t}\right),
$$

uniformly for $t \in \mathbb{R}$ and (38) hold.

Now

$$
\begin{aligned}
& N\left(t+\alpha_{k}\right) \\
& =\int_{0}^{t+\alpha_{k}} U\left(t+\alpha_{k}\right) P U^{-1}(s) n\left(s, x(s), x_{s}\right) \circ \mathrm{d} W(s) \\
& =\int_{0}^{t} U\left(t+\alpha_{k}\right) P U^{-1}\left(s+\alpha_{k}\right) \\
& \quad \times n\left(s+\alpha_{k}, x\left(s+\alpha_{k}\right), x_{s+\alpha_{k}}\right) \circ \mathrm{d} W\left(s+\alpha_{k}\right) \\
& =\int_{0}^{t} U\left(t+\alpha_{k}\right) P U^{-1}\left(s+\alpha_{k}\right) \\
& \quad \times n\left(s+\alpha_{k}, x\left(s+\alpha_{k}\right), x_{s+\alpha_{k}}\right) \circ \mathrm{d} \widetilde{W}(s) .
\end{aligned}
$$

Using Itô's isometry identity, one obtains

$$
\begin{aligned}
& \mathbb{E}\left\|N\left(t+\alpha_{k}\right)\right\|^{2} \\
& =\int_{0}^{t} \mathbb{E}\left\|U\left(t+\alpha_{k}\right) P U^{-1}\left(s+\alpha_{k}\right) n\left(s+\alpha_{k}, x\left(s+\alpha_{k}\right), x_{s+\alpha_{k}}\right)\right\|^{2} \mathrm{~d} s .
\end{aligned}
$$

From (38), (43), (45), and Lebesgue's control convergence theorem, one sees that

$$
T_{\alpha} N(t)=\int_{0}^{t} V(t) P^{*} V^{-1}(s) n_{1}\left(s, x(s), x_{s}\right) \mathrm{d} s
$$

uniformly for $t \in \mathbb{R}$. Hence $N(t) \in A P\left(\mathbb{R}, L^{2}(\mathbb{P}, \mathbb{U})\right)$.
Next, let us show that $M(t) \in C_{0}\left(\mathbb{R}, L^{2}(\mathbb{P}, \mathbb{H})\right)$. In fact

$\mathbb{E}\|M(t)\|^{2}$

$$
\begin{aligned}
= & \mathbb{E}\left\|\int_{0}^{t} U(t) P U^{-1}(s) m\left(s, x(s), x_{s}\right) \circ \mathrm{d} W(s)\right\|^{2} \\
= & \mathbb{E}\left(\left\|\int_{0}^{t} U(t) P U^{-1}(s) m\left(s, x(s), x_{s}\right) \circ \mathrm{d} \widetilde{W}(s)\right\|\right)^{2} \\
\leq & \beta^{2} \int_{0}^{t} \exp (-2 \alpha(t-s)) \\
& \quad \times \mathbb{E}\left\|m\left(s+\tau, x(s+\tau), x_{s+\tau}\right)-m\left(s, x(s), x_{s}\right)\right\|^{2} \mathrm{~d} s \\
\leq & \beta^{2}\left(\int_{0}^{t} \exp (-2 \alpha(t-s) \mathrm{d} s)\right) \\
& \times \sup _{s \in \mathbb{R}} \mathbb{E}\left\|m\left(s+\tau, x(s+\tau), x_{s+\tau}\right)-m\left(s, x(s), x_{s}\right)\right\|^{2} \\
= & \frac{\beta^{2}}{2 \alpha}\left(1-e^{-2 \alpha t}\right) \\
& \times \sup _{s \in \mathbb{R}} \mathbb{E}\left\|m\left(s+\tau, x(s+\tau), x_{s+\tau}\right)-m\left(s, x(s), x_{s}\right)\right\|^{2},
\end{aligned}
$$

where we make extensive use of the Itô isometry identity and the properties of $\widetilde{W}$ defined by $\widetilde{W} \equiv W(s+\tau)-W(\tau)$ for each $s$. Note that $\widetilde{W}$ is also a Brownian motion and has the same distribution as $W$.

Since $m \in C_{0}\left(\mathbb{R} \times L^{2}(\mathbb{P}, \mathbb{H}), L^{2}(\mathbb{P}, \mathbb{W})\right)$, we deduce that $\lim _{t \rightarrow+\infty} \mathbb{E}\|M(t)\|^{2}=0$.

Therefore, $\Psi^{1} x(\cdot)$ is quadratic-mean asymptotically almost periodic.

Similar to the proof given for $\Psi^{1} x(\cdot)$, one can prove that $\Psi^{2} x(\cdot), \Psi^{3} x(\cdot)$, and $\Psi^{4} x(\cdot)$ are quadratic-mean asymptotically almost periodic.

Thus, $B_{2} x$ is quadratic-mean asymptotically almost periodic whenever $x \in A A P\left(\mathbb{R}, L^{2}(\mathbb{P}, \mathbb{U} \mathbb{U})\right.$ ).

In view of the above, it is clear that $\mathbf{L}$ maps $\operatorname{AAP}\left(\mathbb{R}, L^{2}(\mathbb{P}, \mathbb{Q})\right.$ into itself. The proof of Lemma 15 is complete.

Lemma 16. Assume that conditions (H1)-(H3) are satisfied. Then the operator $\mathbf{L}$ is a contraction providing $\Lambda=(8 \beta / \alpha)$ $\sqrt{F_{l}+\alpha H_{l} / 2}<1$.

Proof. Consider

$\|(\mathbf{L} x) t-(\mathbf{L} y) t\|$

$\leq \| \int_{-\infty}^{+\infty} G(t, s)\left[F\left(s, x(s), x_{s}\right)-F\left(s, y(s), x_{s}\right)\right] \mathrm{d} s$

$+\int_{-\infty}^{+\infty} G(t, s)\left[H\left(s, x(s), x_{s}\right)-H\left(s, y(s), x_{s}\right)\right] \circ \mathrm{d} W(s) \|$

$\leq\left\|\int_{-\infty}^{+\infty} G(t, s)\left[F\left(s, x(s), x_{s}\right)-F\left(s, y(s), x_{s}\right)\right] \mathrm{d} s\right\|$ 
$+\left\|\int_{-\infty}^{+\infty} G(t, s)\left[H\left(s, x(s), x_{s}\right)-H\left(s, y(s), x_{s}\right)\right] \circ \mathrm{d} W(s)\right\|$ $:=\left\|C_{1}\right\|+\left\|C_{2}\right\|$.

Since $\left(\sum_{i=1}^{n} a_{i}\right)^{2}<n \sum_{i=1}^{n} a_{i}^{2}$, one can write

$$
\mathbb{E}\|(\mathbf{L} x) t-(\mathbf{L} y) t\|^{2} \leq 2 \mathbb{E}\left\|C_{1}\right\|^{2}+2 \mathbb{E}\left\|C_{2}\right\|^{2} .
$$

Combining (30), one can write

$\left\|C_{1}\right\|$

$$
\begin{aligned}
& =\left\|\int_{-\infty}^{\infty} G(t, s)\left[F\left(s, x(s), x_{s}\right)-F\left(s, y(s), x_{s}\right)\right] \mathrm{d} s\right\| \\
& =\| \int_{0}^{t} U(t) P U^{-1}(s)\left[F\left(s, x(s), x_{s}\right)-F\left(s, y(s), x_{s}\right)\right] \mathrm{d} s \\
& \quad-\int_{\max \{t, 0\}}^{+\infty} U(t)(I-P) U^{-1}(s) \\
& \quad \times\left[F\left(s, x(s), x_{s}\right)-F\left(s, y(s), x_{s}\right)\right] \mathrm{d} s \\
& \quad+\int_{-\infty}^{0} U(t) Q U^{-1}(s)\left[F\left(s, x(s), x_{s}\right)-F\left(s, y(s), x_{s}\right)\right] \mathrm{d} s \\
& \quad U(t)(I-Q) U^{-1}(s) \\
& \left.\quad+\int_{t}^{\min \{t, 0\}} e^{-\alpha(s-t)}+\int_{-\infty}^{\min \{t, 0\}} e^{-\alpha(t-s)}\right] \\
& \leq \beta\left[\int_{0}^{t} e^{-\alpha(t-s)}+\int_{\max \{t, 0\}}^{+\infty} e^{-\alpha(s-t)}\right. \\
& \left.\quad x(s), x_{s}\right)-F\left(s, y(s), x_{s}\right) \| \mathrm{d} s .
\end{aligned}
$$

Thus,

\section{$\mathbb{E}\left\|C_{1}\right\|^{2}$}

$$
\begin{aligned}
& \leq 4 \beta^{2} \mathbb{E}\left(\int_{0}^{t} e^{-\alpha(t-s)}\left\|F\left(s, x(s), x_{s}\right)-F\left(s, y(s), x_{s}\right)\right\| \mathrm{d} s\right)^{2} \\
& +4 \beta^{2} \mathbb{E}\left(\int_{\max \{t, 0\}}^{+\infty} e^{-\alpha(s-t)}\left\|F\left(s, x(s), x_{s}\right)-F\left(s, y(s), x_{s}\right)\right\| \mathrm{d} s\right)^{2} \\
& +4 \beta^{2} \mathbb{E}\left(\int_{t}^{0} e^{-\alpha(s-t)}\left\|F\left(s, x(s), x_{s}\right)-F\left(s, y(s), x_{s}\right)\right\| \mathrm{d} s\right)^{2} \\
& +4 \beta^{2} \mathbb{E}\left(\int_{-\infty}^{\min \{t, 0\}} e^{-\alpha(t-s)}\left\|F\left(s, x(s), x_{s}\right)-F\left(s, y(s), x_{s}\right)\right\| \mathrm{d} s\right)^{2} \\
& :=U_{1}+U_{2}+U_{3}+U_{4} .
\end{aligned}
$$

We first evaluate $U_{1}$ as follows:

$U_{1}$

$$
\begin{aligned}
& =4 \beta^{2} \mathbb{E}\left(\int_{0}^{t} e^{-\alpha(t-s)}\left\|F\left(s, x(s), x_{s}\right)-F\left(s, y(s), x_{s}\right)\right\| \mathrm{d} s\right)^{2} \\
& \leq 4 \beta^{2} \mathbb{E}\left[\left(\int_{0}^{t} e^{-\alpha(t-s)} \mathrm{d} s\right)\right. \\
& \left.\quad \times\left(\int_{0}^{t} e^{-\alpha(t-s)}\left\|F\left(s, x(s), x_{s}\right)-F\left(s, y(s), x_{s}\right)\right\|^{2} \mathrm{~d} s\right)\right] \\
& \leq 4 \beta^{2}\left(\int_{0}^{t} e^{-\alpha(t-s)} \mathrm{d} s\right) \\
& \times\left(\int_{0}^{t} e^{-\alpha(t-s)} \mathbb{E}\left\|F\left(s, x(s), x_{s}\right)-F\left(s, y(s), x_{s}\right)\right\|^{2} \mathrm{~d} s\right) \\
& \leq 4 \beta^{2} F_{l}\left(\int_{0}^{t} e^{-\alpha(t-s)} \mathrm{d} s\right)^{2} \\
& \quad \times \sup _{s \in \mathbb{R}}\left(\|x(s)-y(s)\|_{L^{2}(\mathbb{P}, \mathbb{H})}^{2}+\|x(s)-y(s)\|_{C}^{2}\right) \\
& \leq \frac{8 \beta^{2} F_{l}}{\alpha^{2}}\left[1-e^{-\alpha t}\right]\|x(\cdot)-y(\cdot)\|_{\infty}^{2} \leq \frac{8 \beta^{2} F_{l}}{\alpha^{2}}\|x(\cdot)-y(\cdot)\|_{\infty}^{2} .
\end{aligned}
$$

Similar to the discussion given for $U_{1}$, for $U_{2}, U_{3}$, and $U_{4}$, one has

$$
\begin{aligned}
& U_{2} \\
& =4 \beta^{2} \mathbb{E}\left(\int_{\max \{t, 0\}}^{+\infty} e^{-\alpha(s-t)}\left\|F\left(s, x(s), x_{s}\right)-F\left(s, y(s), x_{s}\right)\right\| \mathrm{d} s\right)^{2} \\
& \leq \frac{8 \beta^{2} F_{l}}{\alpha^{2}}\|x(\cdot)-y(\cdot)\|_{\infty}^{2}, \\
& U_{3} \leq \frac{8 \beta^{2} F_{l}}{\alpha^{2}}\|x(\cdot)-y(\cdot)\|_{\infty}^{2}, \quad U_{4} \leq \frac{8 \beta^{2} F_{l}}{\alpha^{2}}\|x(\cdot)-y(\cdot)\|_{\infty}^{2} .
\end{aligned}
$$

Then,

$$
\mathbb{E}\left\|C_{1}\right\|^{2} \leq \frac{32 \beta^{2} F_{l}}{\alpha^{2}}\|x(\cdot)-y(\cdot)\|_{\infty}^{2}
$$

$$
\text { Next, we evaluate } \mathbb{E}\left\|C_{2}\right\|^{2} \text { as follows: }
$$

\section{$\mathbb{E}\left\|C_{2}\right\|^{2}$}

$$
\begin{aligned}
& =\mathbb{E}\left\|\int_{-\infty}^{\infty} G(t, s)\left[H\left(s, x(s), x_{s}\right)-H\left(s, y(s), x_{s}\right)\right] \circ \mathrm{d} W(s)\right\|^{2} \\
& \leq 4 \mathbb{E} \| \int_{0}^{t} U(t) P U^{-1}(s) \\
& \quad \times\left[H\left(s, x(s), x_{s}\right)-H\left(s, y(s), x_{s}\right)\right] \circ \mathrm{d} W(s) \|^{2}
\end{aligned}
$$




$$
\begin{aligned}
& +4 \mathbb{E} \| \int_{\max \{t, 0\}}^{+\infty} U(t)(I-P) U^{-1}(s) \\
& \times\left[H\left(s, x(s), x_{s}\right)-H\left(s, y(s), x_{s}\right)\right] \circ \mathrm{d} W(s) \|^{2} \\
& +4 \mathbb{E} \| \int_{t}^{0} U(t) Q U^{-1}(s) \\
& \times\left[H\left(s, x(s), x_{s}\right)-H\left(s, y(s), x_{s}\right)\right] \circ \mathrm{d} W(s) \|^{2} \\
& +4 \mathbb{E} \| \int_{-\infty}^{\min \{t, 0\}} U(t)(I-Q) U^{-1}(s) \\
& \times\left[H\left(s, x(s), x_{s}\right)-H\left(s, y(s), x_{s}\right)\right] \circ \mathrm{d} W(s) \|^{2} \\
& :=V_{1}+V_{2}+V_{3}+V_{4} \text {. }
\end{aligned}
$$

As for the first term $V_{1}$, using Itô's isometry identity, one obtains

$$
\begin{aligned}
& V_{1} \\
& =4 \mathbb{E} \| \int_{0}^{t} U(t) P U^{-1}(s) \\
& \quad \times\left[H\left(s, x(s), x_{s}\right)-H\left(s, y(s), x_{s}\right)\right] \circ \mathrm{d} W(s) \|^{2} \\
& =4 \mathbb{E}\left[\int_{0}^{t}\left\|U(t) P U^{-1}(s)\left[H\left(s, x(s), x_{s}\right)-H\left(s, y(s), x_{s}\right)\right]\right\|^{2} \mathrm{~d} s\right] \\
& \leq 4 \beta^{2} \int_{0}^{t} e^{-2 \alpha(t-s)} \mathbb{E}\left\|H\left(s, x(s), x_{s}\right)-H\left(s, y(s), x_{s}\right)\right\|^{2} \mathrm{~d} s \\
& \leq 4 \beta^{2} H_{l}\left(\int_{0}^{t} e^{-2 \alpha(t-s)} \mathrm{d} s\right) \\
& \quad \times \sup _{s \in \mathbb{R}}\left(\|x(s)-y(s)\|_{L^{2}(\mathbb{P}, \mathbb{H})}^{2}+\|x(s)-y(s)\|_{C}^{2}\right) \\
& \leq \frac{8 \beta^{2} H_{l}}{\alpha}\left[1-e^{-2 \alpha t}\right]\|x(\cdot)-y(\cdot)\|_{\infty}^{2} \leq \frac{8 \beta^{2} H_{l}}{\alpha}\|x(\cdot)-y(\cdot)\|_{\infty}^{2} .
\end{aligned}
$$

Similarly, one can evaluate the second term $V_{2}$, third term $V_{3}$, and the fourth term $V_{4}$ of the right-hand side, respectively:

$$
\begin{aligned}
& V_{2} \\
& =4 \mathbb{E} \| \int_{\max \{t, 0\}}^{+\infty} U(t) P U^{-1}(s) \\
& \quad \times\left[H\left(s, x(s), x_{s}\right)-H\left(s, y(s), x_{s}\right)\right] \circ \mathrm{d} W(s) \|^{2}
\end{aligned}
$$

$$
\begin{gathered}
\leq \frac{8 \beta^{2} H_{l}}{\alpha}\|x(\cdot)-y(\cdot)\|_{\infty}^{2}, \\
V_{3} \leq \frac{8 \beta^{2} H_{l}}{\alpha}\|x(\cdot)-y(\cdot)\|_{\infty}^{2}, \\
V_{4} \leq \frac{8 \beta^{2} H_{l}}{\alpha}\|x(\cdot)-y(\cdot)\|_{\infty}^{2} .
\end{gathered}
$$

Therefore,

$$
\mathbb{E}\left\|C_{2}\right\|^{2} \leq \frac{32 \beta^{2} H_{l}}{\alpha}\|x(\cdot)-y(\cdot)\|_{\infty}^{2} .
$$

Thus, by combing (54) and (58), it follows that

$$
\mathbb{E}\|(\mathbf{L} x)(t)-(\mathbf{L} y)(t)\|^{2} \leq \frac{64 \beta^{2}}{\alpha^{2}}\left[F_{l}+\frac{\alpha H_{l}}{2}\right]\|x(\cdot)-y(\cdot)\|_{\infty}^{2},
$$

which implies that

$$
\begin{aligned}
& \|(\mathbf{L} x)(t)-(\mathbf{L} y)(t)\|_{\infty} \leq \frac{8 \beta}{\alpha} \sqrt{F_{l}+\frac{\alpha H_{l}}{2}}\|x(\cdot)-y(\cdot)\|_{\infty} \\
& =\Lambda\|x-y\|_{\infty} .
\end{aligned}
$$

Since $\Lambda<1$, by (60), we know that $\mathbf{L}$ is a contraction mapping. The proof of Lemma 16 is complete.

Hence, combining Lemmas 15 and 16 and the contraction mapping principle, $\mathbf{L}$ has a unique fixed point $x(t)$, which is obviously the unique quadratic-mean asymptotically almost periodic mild solution of (26).

This completes the proof of Theorem 14 due to Lemmas 15 and 16.

Remark 17. If the conditions of the main result of [14] and (H1) and (H2) hold, (26) admits exponential trichotomy with projections $P, Q$ and $P Q=0$; hence system (26) has a unique quadratic-mean asymptotically almost periodic mild solution. So our main result improves the main result of [14].

\section{Example}

The deterministic nonlinear Duffing-van der Pol equation

$$
\ddot{y}=a y+b \dot{y}+r y^{3}+d y^{2} \dot{y}+s \dot{y}^{3}, \quad a, b, r, d, s \in \mathbb{R},
$$

has become a paradigm for mathematicians, physicists, and engineers. There are numerous physical and engineering problems whose dynamics are described by (61) for some parameter values.

In particular, for $r=s=0$ one obtains the van der Pol equation and for $d=s=0$ one obtains the Duffing equation.

As an example, we consider the following functional differential equation for small $\epsilon_{1}$ and $\epsilon_{2}$ :

$$
\ddot{y}=-(a+b p(t)) y+\epsilon_{1} f\left(t, y, y_{t}\right)+\epsilon_{2} h\left(t, y, y_{t}\right) \dot{y} \text {. }
$$


It is easy to see that (61) is the general form of the Duffingvan der Pol equation (61). By perturbing the remaining parameters and the right-hand side of (62) by real or white noise, one arrives at noise, more specifically the random (for real noise) or stochastic (for white noise) functional differential equation, respectively:

$$
\ddot{y}=-(a+b p(t)) y+\epsilon_{1} f\left(t, y, y_{t}\right)+\epsilon_{2} \xi_{2}(t) h\left(t, y, y_{t}\right) \dot{y},
$$

where $s_{2}$ is intensity parameter, functions $f \in A A P(\mathbb{R} \times$ $\left.L^{2}(\mathbb{P}, \mathbb{R}) \times C ; L^{2}(\mathbb{P}, \mathbb{R})\right), h \in A A P\left(\mathbb{R} \times L^{2}(\mathbb{P}, \mathbb{R}) \times\right.$ $C ; L^{2}\left(\mathbb{P}, \mathscr{L}\left(L^{2}(\mathbb{P}, \mathbb{R})\right)\right)$, and $\xi(t)=\left(\xi_{1}(t), \xi_{2}(t)\right)=\left(0, \xi_{2}(t)\right)$ is a stationary process or white noise. As a first order system for $x=\left(x_{1}, x_{2}\right)^{T}=(y, \dot{y})^{T},(63)$ takes the form

$$
\begin{array}{r}
\mathrm{d} x(t)=\left[A(t) x(t)+F\left(t, x, x_{t}\right)\right] \mathrm{d} t+H\left(t, x, x_{t}\right) \circ \mathrm{d} W(t), \\
t \in \mathbb{R},
\end{array}
$$

where

$$
\begin{gathered}
A(t)=\left(\begin{array}{cc}
0 & 1 \\
-(a+b p(t)) & 0
\end{array}\right), \\
F\left(t, x, x_{t}\right)=\left(\begin{array}{c}
0 \\
\epsilon_{1} f\left(t, x_{1}, x_{1 t}\right)
\end{array}\right), \\
H\left(t, x, x_{t}\right)=\left(\begin{array}{c}
0 \\
\epsilon_{2} h\left(t, x_{1}, x_{1 t}\right) x_{2}
\end{array}\right) .
\end{gathered}
$$

Let $x=A A P\left(\mathbb{R} ; L^{2}\left(\mathbb{P}, \mathbb{R}^{2}\right)\right)$. Suppose that vector functions $F$ and $H$ satisfy the inequality conditions of (H1) and $(\mathrm{H} 2)$ in Theorem 14. We see that when (i) $b \geq 0, a+$ $\sup _{t \in \mathbb{R}} p(t)<0$ or (ii) $b<0, a+\inf _{t \in \mathbb{R}} p(t)>0$ hold, $\mathrm{d} x / \mathrm{d} t=A(t) x(t)$ admits exponential trichotomy, as in [24, 25]. That is, there exist constants $\alpha$ and $\beta$ such that (16) holds. When $(8 \beta / \alpha) \sqrt{\epsilon_{1} F_{l}+\alpha \epsilon_{2} H_{l} / 2}<1$, the other conditions of Theorem 14 are satisfied. So (63) has a unique quadratic-mean asymptotically almost periodic mild solution.

Remark 18. The linear system of the system (63) admits exponential trichotomy with projections $P, Q$ and $P Q=0$; hence the main result of [14] cannot ensure the existence and uniqueness of asymptotically almost periodic mild solution of (63). It is obvious that the condition that "the family $\{A(t)$ : $t \in \mathbb{R}\}$ of operators in $\mathbb{R}$ generates an exponentially stable semigroup $\{T(t)\}_{t \geq 0}$ " cannot be satisfied. So the main result of $[18,19,21]$ cannot also ensure the existence of almost periodic type mild solution of (63).

\section{Conclusions}

In this paper, a new criterion ensuring the existence and uniqueness of the quadratic-mean asymptotically almost periodic mild solutions for a class of stochastic functional differential equations is presented. The condition of being uniformly exponentially stable of the linear operator is essentially removed, only using its exponential trichotomy, which reflects a deeper analysis of the behavior of solutions of the system. In this case the asymptotic behavior is described through the splitting of the main space into stable, unstable, and central subspaces at each point from the flow's domain. An example is also given to illustrate our results.

\section{Conflict of Interests}

The authors declare that there is no conflict of interests regarding the publication of this paper.

\section{Acknowledgments}

The authors wish to thank the anonymous reviewers whose suggestions and corrections have significantly improved the presentation of this paper. This work was supported by the National Natural Science Foundation of China (no. 11161051) and the Scientific Research Foundation of Guangxi Education Office of China (no. 2014).

\section{References}

[1] A. M. Fink, Almost Periodic Differential Equations, vol. 377 of Lecture Notes in Mathematics, Springer, New York, NY, USA, 1974.

[2] C. Zhang, Almost Periodic Type Functions and Ergodicity, Science Press, Beijing, China, 2006.

[3] P. H. Bezandry, "Existence of almost periodic solutions to some functional integro-differential stochastic evolution equations," Statistics \& Probability Letters, vol. 78, no. 17, pp. 2844-2849, 2008.

[4] E. Slutsky, "Sur les fonctions aléatoires presque périodiques et sur la decomposition des functions aléatoires," in Actualités Scientifiques et Industrielles, vol. 738, pp. 33-55, Herman, Paris, France, 1938.

[5] M. Udagawa, "Asymptotic properties of distributions of some functionals of random variables," Reports of Statistical Application Research. Union of Japanese Scientists and Engineers, vol. 2, no. 2-3, pp. 1-98, 1952.

[6] T. Kawata, "Almost periodic weakly stationary processes", in Statistics and Probability: Essays in Honor of C. R. Rao, pp. 383396, North-Holland, New York, NY, USA, 1982.

[7] R. J. Swift, "Almost periodic harmonizable processes," Georgian Mathematical Journal, vol. 3, no. 3, pp. 275-292, 1996.

[8] P. H. Bezandry and T. Diagana, "Square-mean almost periodic solution to some nonautonomous stochastic differential equations," Electronic Journal of Differential Equations, vol. 2007, no. 117, pp. 1-10, 2007.

[9] J. Luo, "Stochastically bounded solutions of a nonlinear stochastic differential equations," Journal of Computational and Applied Mathematics, vol. 196, no. 1, pp. 87-93, 2006.

[10] W. M. Ruess, "Asymptotic almost periodicity and motions of semigroups of operators," Linear Algebra and Its Applications, vol. 84, pp. 335-351, 1986.

[11] W. M. Ruess and V. Q. Phong, "Asymptotically almost periodic solutions of evolution equations in Banach spaces," Journal of Differential Equations, vol. 122, no. 2, pp. 282-301, 1995.

[12] W. Arendt and C. J. K. Batty, "Asymptotically almost periodic solutions of inhomogeneous Cauchy problems on the half-line," 
Bulletin of the London Mathematical Society, vol. 31, no. 3, pp. 291-304, 1999.

[13] W. Arendt and C. J. K. Batty, "Almost periodic solutions of firstand second-order Cauchy problems," Journal of Differential Equations, vol. 137, no. 2, pp. 363-383, 1997.

[14] J. Cao, Q. Yang, Z. Huang, and Q. Liu, "Asymptotically almost periodic solutions of stochastic functional differential equations," Applied Mathematics and Computation, vol. 218, no. 5, pp. 1499-1511, 2011.

[15] J. Cao, Q. Yang, and Z. Huang, "On almost periodic mild solutions for stochastic functional differential equations," Nonlinear Analysis: Real World Applications, vol. 13, no. 1, pp. 275-286, 2012.

[16] H. Huang and J. Cao, "Exponential stability analysis of uncertain stochastic neural networks with multiple delays," Nonlinear Analysis: Real World Applications, vol. 8, no. 2, pp. 646-653, 2007.

[17] J. Cao, Q. Yang, and Z. Huang, "Existence and exponential stability of almost automorphic mild solutions for stochastic functional differential equations," Stochastics, vol. 83, no. 3, pp. 259-275, 2011.

[18] P. H. Bezandry and T. Diagana, "Existence of almost periodic solutions to some stochastic differential equations," Applicable Analysis, vol. 86, no. 7, pp. 819-827, 2007.

[19] P. H. Bezandry and T. Diagana, "Existence of quadratic-mean almost periodic solutions to some stochastic hyperbolic differential equations," Electronic Journal of Differential Equations, vol. 2009, no. 111, pp. 1-14, 2009.

[20] P. H. Bezandry and T. Diagana, "Existence of square-mean almost periodic mild solutions to some nonautonomous stochastic second-order differential equations," Electronic Journal of Differential Equations, vol. 2010, no. 124, pp. 1-25, 2010.

[21] M. Fu and Z. Liu, "Square-mean almost automorphic solutions for some stochastic differential equations," Proceedings of the American Mathematical Society, vol. 138, no. 10, pp. 3689-3701, 2010.

[22] Y. Liu and A. Liu, "Almost periodic solutions for a class of stochastic differential equations," Journal of Computational and Nonlinear Dynamics, vol. 8, no. 4, Article ID 041002, 6 pages, 2013.

[23] S. Elaydi and O. Hájek, "Exponential trichotomy of differential systems," Journal of Mathematical Analysis and Applications, vol. 129, no. 2, pp. 362-374, 1988.

[24] J. Hong, R. Obaya, and A. Sanz, "Existence of a class of ergodic solutions implies exponential trichotomy," Applied Mathematics Letters, vol. 12, no. 4, pp. 43-45, 1999.

[25] L. Barreira and C. Valls, "Lyapunov functions for trichotomies with growth rates," Journal of Differential Equations, vol. 248, no. 1, pp. 151-183, 2010. 


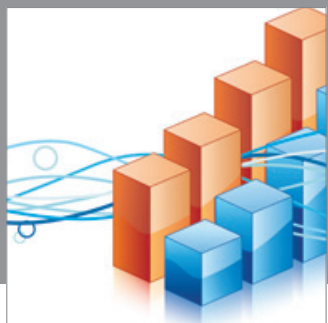

Advances in

Operations Research

mansans

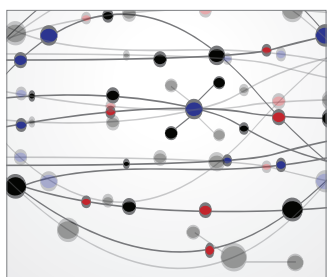

The Scientific World Journal
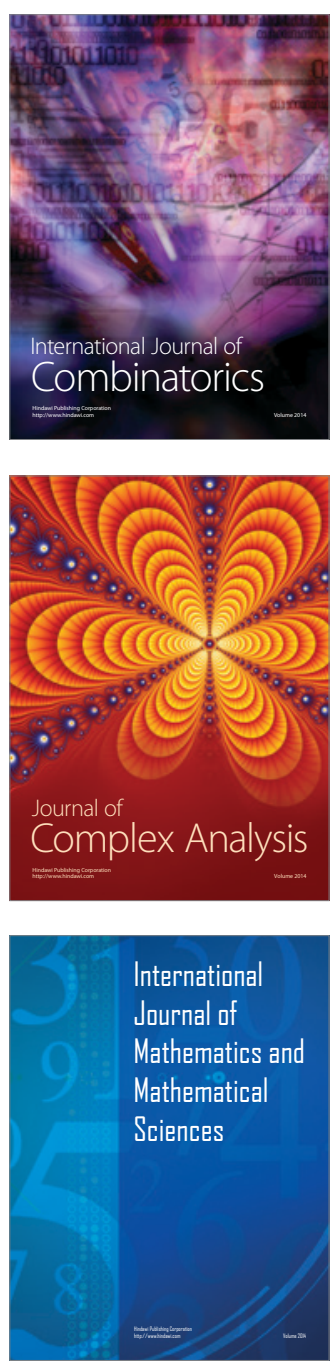
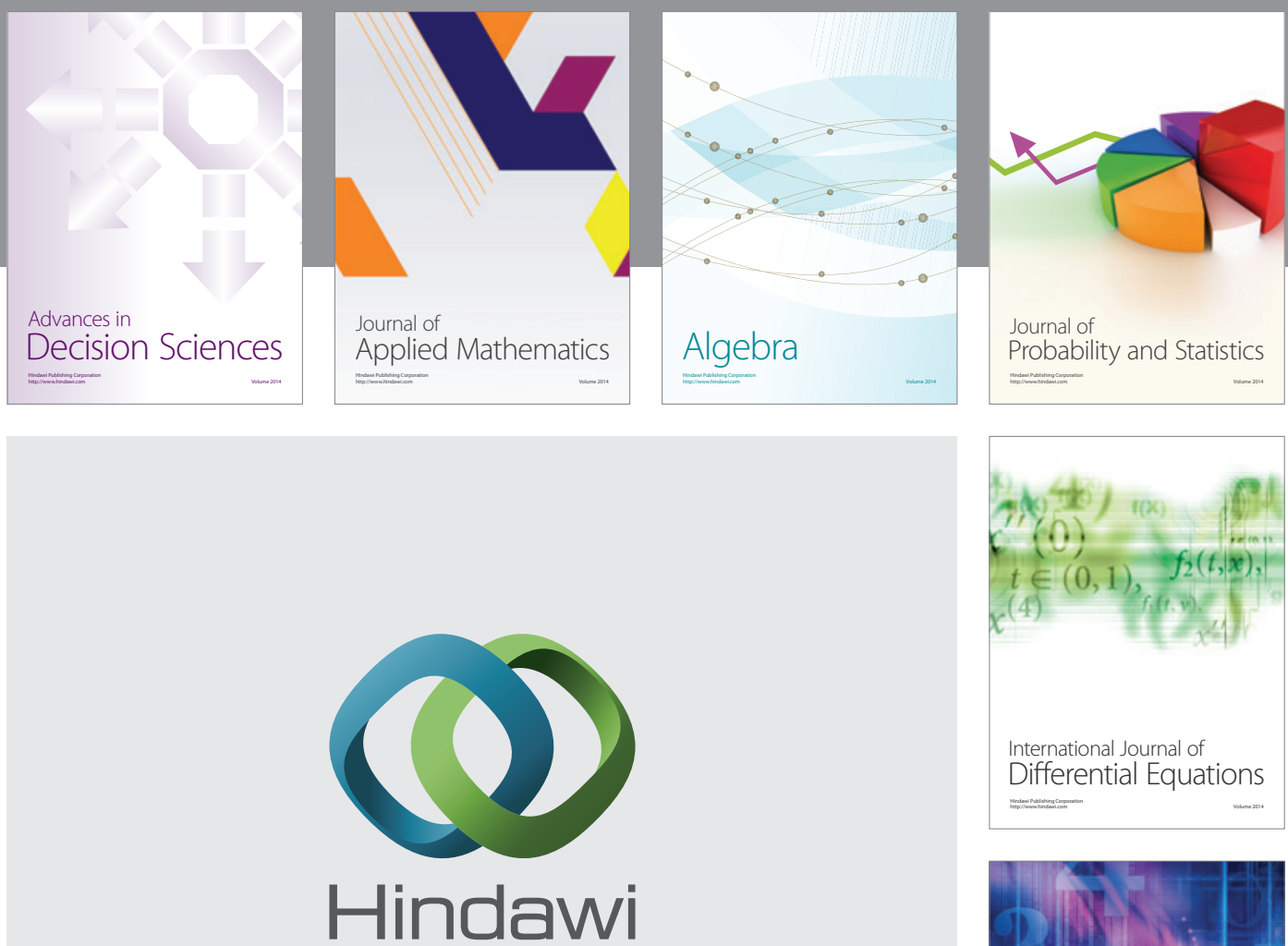

Submit your manuscripts at http://www.hindawi.com
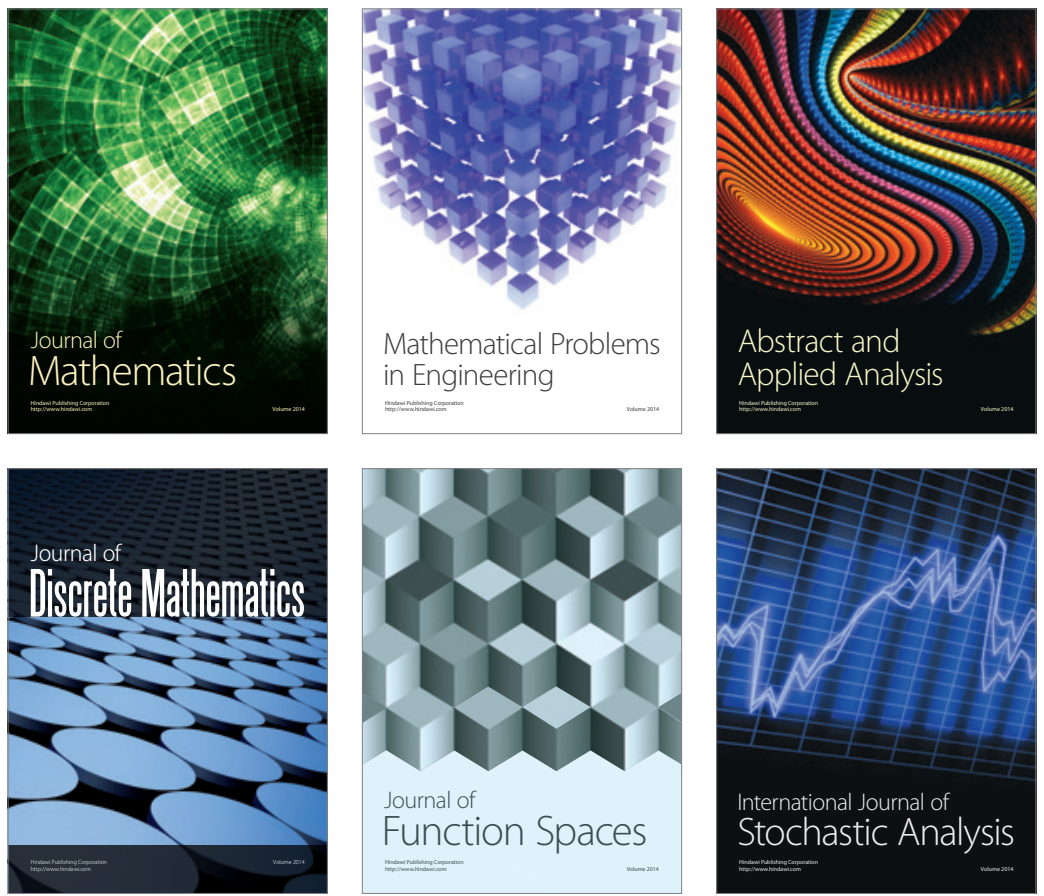

Journal of

Function Spaces

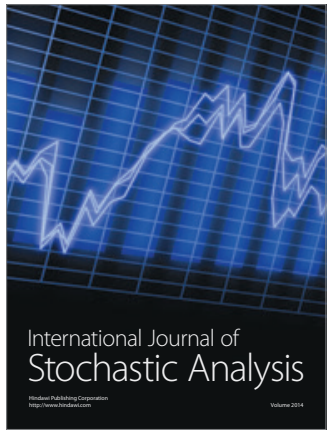

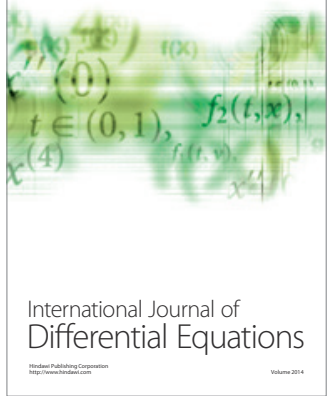
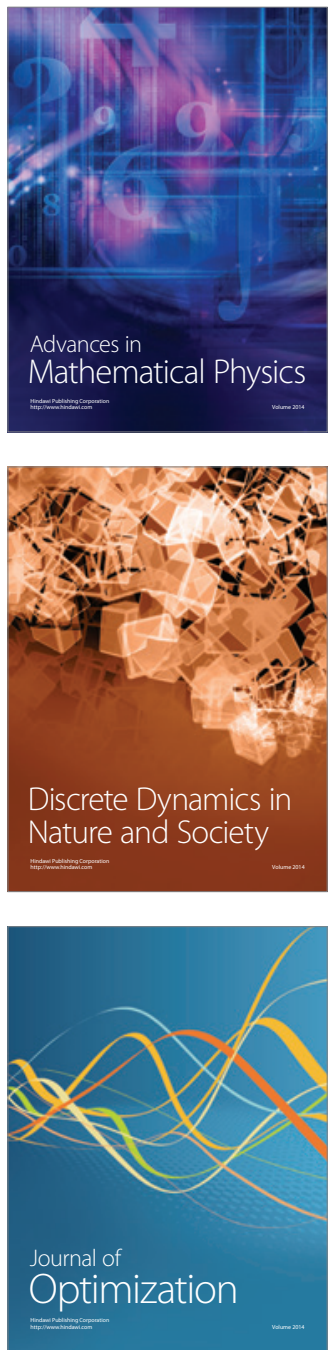